\title{
The Role of Massive Transfusion Protocols in Obstetrics
}

\author{
Luis D. Pacheco, MD ${ }^{1,2}$ George R. Saade, MD ${ }^{1}$ Maged M. Costantine, MD ${ }^{1} \quad$ Steven L. Clark, MD ${ }^{3}$ \\ Gary D.V. Hankins, MD ${ }^{1}$
}
${ }^{1}$ Division of Maternal-Fetal Medicine, Department of Obstetrics and Gynecology, The University of Texas Medical Branch, Galveston, Texas
2 Division of Surgical Critical Care, Department of Anesthesiology, The University of Texas Medical Branch, Galveston, Texas
${ }^{3}$ Women and Newborns Clinical Program, Hospital Corporation of America, Salt Lake City, Utah

Am J Perinatol 2013;30:1-4.

\begin{abstract}
Address for correspondence and reprint requests Luis D. Pacheco, MD, The University of Texas Medical Branch at Galveston, 301 University Blvd., Galveston, TX 77555-0587 (e-mail: Idpachec@utmb.edu).
\end{abstract}

\begin{abstract}
Keywords

- obstetric hemorrhage

- massive transfusion

- hemostasis

Obstetric hemorrhage is a leading cause of maternal mortality worldwide. New concepts involving the pathophysiology of hemorrhage have been described and include early activation of both the protein $\mathrm{C}$ and fibrinolytic pathways. New tendencies in hemorrhage treatment include the use of hemostatic resuscitation. Massive transfusion protocols involve the early utilization of blood products and limit the traditional approach of early massive crystalloid based resuscitation. The evidence behind hemostatic resuscitation is still limited.
\end{abstract}

Hemorrhage constitutes the most common form of shock in obstetric practice and remains a major cause of maternal mortality worldwide. In 2005, hemorrhage was the third leading cause of maternal death due to obstetric factors in the United States. ${ }^{1}$

Current trends in obstetric practice (increased cesarean section rate and decreased vaginal birth after cesarean section) have resulted in increased prevalence of placental anomalies (both placenta previa and accreta), placing peripartum hemorrhage as one of the most important potential causes for maternal mortality. ${ }^{2}$

Recent literature has suggested that alternative pathways could play a key role in the pathophysiology of massive hemorrhage. Also, current transfusion guidelines have been questioned, and limited evidence suggests that early aggressive blood product replacement (hemostatic resuscitation) in the setting of massive hemorrhage can improve outcomes. ${ }^{3}$ We have recently published an article regarding the cuttingedge advances in the medical management of obstetric hemorrhage elsewhere. ${ }^{4}$ This review focuses specifically on the role of massive transfusion protocols.

Despite limited prospective data, many level I trauma centers and obstetric units in the United States have adopted the use of massive transfusion protocols to treat patients with severe hemorrhage. ${ }^{5}$ These protocols, if adopted, require a multidisciplinary approach involving obstetricians, anesthesiologists, hematologists, and blood bank personnel. Once the involved surgeon calls the blood bank and activates the protocol, blood bank personnel will provide blood products (packed red blood cells [PRBC], fresh frozen plasma [FFP], cryoprecipitate, platelets) at a predefined ratio without questioning the liberation of such products based on laboratory values. The protocol will continue until it is inactivated by the surgeon either because bleeding has been controlled or the patient dies. In this article, we will discuss the advantages and disadvantages of massive transfusion protocols and will propose a protocol for use during obstetric hemorrhage. received

January 30, 2012

accepted after revision

March 16, 2012

published online

July 26, 2012
Copyright $\odot 2013$ by Thieme Medical Publishers, Inc., 333 Seventh Avenue, New York, NY 10001, USA.

Tel: +1(212) 584-4662.
DOI http://dx.doi.org/ 10.1055/s-0032-1322511. ISSN 0735-1631. 


\section{New Concepts in Acute Coagulopathy and Hemostatic Resuscitation}

Classically, resuscitation in hemorrhage has been centered on administration of crystalloids and PRBC. Use of other blood products like FFP, cryoprecipitate, and platelets is indicated in the presence of abnormal laboratory values (e.g., platelet count $<50,000 / \mathrm{mm}^{3}$, fibrinogen $<100 \mathrm{mg} / \mathrm{dL}$, prothrombin time, or activated partial thromboplastin time $>1.5 \times$ normal). These current transfusion guidelines fail to prevent early coagulopathy in cases of massive bleeding. ${ }^{6}$ Patients with crystalloid/PRBC-based resuscitation will frequently develop dilution of clotting factors and platelets, leading to the so called "dilutional coagulopathy." The latter may be complicated by hypothermia and acidosis, both of which further compound coagulation dysfunction.

Massive crystalloid resuscitation may further worsen bleeding prior to achieving surgical control of hemorrhage by increasing intravascular hydrostatic pressures and dislodging fresh clots at sites of endothelial injury. ${ }^{7}$

The intravascular half-life of crystalloids is 30 to 60 minutes. Excessive amounts of crystalloids have been associated with third spacing, leading to cerebral, cardiac, and pulmonary edema. ${ }^{8}$ Third spacing from massive crystalloidbased resuscitation may also worsen hemodynamics and renal perfusion by increasing intra-abdominal pressures, leading to abdominal compartment syndrome. ${ }^{8}$ Furthermore, recent evidence suggests that excessive administration of crystalloids in the setting of acute kidney injury (not uncommon in massive hemorrhage) results in interstitial kidney edema with renal vein obliteration delaying the recovery of kidney function. ${ }^{9}$ Chlorine-rich fluids (e.g., $0.9 \%$ saline) worsen kidney function, as chloride induces renal vasoconstriction. ${ }^{10}$

Prior to surgical control of hemorrhage, permissive hypotension with systolic blood pressures between 80 and $100 \mathrm{~mm} \mathrm{Hg}$ (resuscitation titrated to a palpable radial pulse) may be optimal to limit ongoing blood loss. ${ }^{7}$ This is a conscious choice to allow hypotension until surgical repair is accomplished. Overzealous crystalloid resuscitation prior to surgical control of the bleeding source should be avoided. Permissive hypotension may be considered in patients with postpartum hemorrhage. However, there are no data regarding permissive hypotension prior to delivery of the fetus, when the risks and benefits need to take into account the effect on uterine perfusion pressure.

Recent data have shown that early coagulopathy may occur prior to hemodilution and before consumption of clotting factors takes place. This mechanism of early coagulopathy has mainly been studied in trauma; however, obstetric hemorrhage may share some of the mechanisms involved. ${ }^{7}$ Early tissue hypoperfusion leads to up-regulation of thrombomodulin on endothelial cells. This receptor interacts with thrombin, leading to activation of the protein $C$ pathway. Protein $C$ is a natural anticoagulant that will irreversibly inhibit factors Va and VIIIa and enhance fibrinolysis through inhibition of plasminogen activator inhibitor $1 .^{11}$ Increased fibrinolytic activity has been described in obstetric hemorrhage second- ary to uterine atony, placental abruption, and accretism. ${ }^{7}$ Early use of antifibrinolytic agents (e.g., epsilon-aminocaproic acid or tranexamic acid) has been shown to decrease bleeding and mortality in trauma victims. ${ }^{12}$ This new concept of coagulopathy has challenged the current resuscitation guidelines, suggesting that early clotting factor replacement and early identification of excessive fibrinolysis with subsequent administration of antifibrinolytic agents could be associated with improved outcomes and decreased transfusion requirements.

Hemostatic resuscitation has emerged as a paradigm shift and an attempt to overcome the previously described shortcomings. The key concept of this approach is to limit the administration of crystalloids and instead make blood products the cornerstone of resuscitation. ${ }^{3}$

In hemostatic resuscitation, PRBC are administered in a $1: 1: 1$ ratio with FFP and platelets. The administration of these products is given regardless of laboratory values. Theoretically, clotting factor dilution and third spacing are minimized with this approach.

Overall, the evidence behind the use of hemostatic resuscitation is limited. Retrospective military and civilian studies have demonstrated absolute mortality reductions between $15 \%$ and $62 \%$ with the use of higher ratios of FFP:PRBC. ${ }^{13,14} \mathrm{~A}$ significant limitation of the available literature on this topic is the presence of survival bias. On average, the median time for obtaining the first unit of PRBC is 18 minutes, as opposed to more than 1 hour for FFP (needs to be thawed). ${ }^{15}$ It is likely that the patients who received FFP were not as sick as those who died before the FFP was available. The need to survive until FFP was available to be included in the group of patients who received it may have biased the analysis in favor of high FFP:PRBC ratios. A limited number of studies have specifically addressed the survivorship bias in high FFP:PRBC studies. When early deaths were excluded, no survival benefit for higher ratios was noted. ${ }^{16}$ A recent review of the literature concluded that there is insufficient evidence to support a survival advantage with a 1:1 plasma-to-PRBC transfusion strategy. ${ }^{5}$ Prospective trials are required to validate the benefit of early FFP and platelet administration.

Depending on the protocol utilized, after a certain number of blood products are administered, if bleeding persists, early administration of recombinant activated factor VII (rFVIIa) is recommended. rFVIla is licensed for use in patients with hemophilia and inhibitory alloantibodies against factors VIII or IX. ${ }^{17}$ It is increasingly being used for off-license indications including trauma, heart surgery after cardiopulmonary bypass, vascular surgery, warfarin reversal, and obstetric hemorrhage. More than $75 \%$ of level I trauma centers in the United States recommend the use of rFVIIa in their massive transfusion protocols. Despite having a very short half-life (2 to 6 hours), concerns about thromboembolism as a complication are real. rFVIla is not a first-line treatment for bleeding, and it will only be effective once major sources of bleeding have been controlled. The use of this product should be combined with best practice use of blood products. ${ }^{18}$ Ideally, the patient should have a platelet count $>50,000 / \mathrm{mm}^{3}$, fibrinogen $>50$ to $100 \mathrm{mg} / \mathrm{dL}$, 
temperature $>32^{\circ} \mathrm{C}, \mathrm{pH}>7.2$, and normal ionized calcium prior to administration. ${ }^{18}$ The normal ionized calcium will facilitate adequate functioning of the clotting cascade.

Randomized controlled trials addressing the use of rFVIIa in massive hemorrhage have found an overall reduction in transfusion requirements or blood loss, but none has reported a survival benefit. ${ }^{5,19}$ Overall, it looks like rFVIIa is effective in limiting the amount of blood products transfused, but data on survival benefit are lacking.

Limited data suggest the use of fibrinogen concentrates in the setting of obstetric hemorrhage. Fibrinogen concentrates will replace fibrinogen levels with a small-volume infusion and with minimal preparation time. ${ }^{20}$

\section{Potential Disadvantages of Massive Transfusion Protocols}

Adoption of massive transfusion protocols will inevitably result in a significant burden for the blood bank personnel. The economic repercussions of utilizing multiple blood products and thawing FFP are also significant. It is of utmost importance that when the transfusion requirements decrease (e.g., hemorrhage control has been achieved), the blood bank be notified to stop further preparation of blood products that would otherwise be wasted.

Early administration of multiple blood products could lead to a higher incidence of transfusion-related complications like transfusion-related acute lung injury and transfusionrelated immunomodulation. ${ }^{21}$ Some argue the opposite, with the rationale that early administration of FFP and platelets achieves hemostasis earlier, thus decreasing the total number of blood products given. ${ }^{6}$

Excessive use of blood products could also increase the risk of transfusion-related infectious diseases, allergic and febrile reactions, transfusion-related acute kidney injury, transfusion-related circulatory overload, and metabolic complications, including hyperkalemia, hypocalcemia, iron overload, and citrate toxicity. ${ }^{22}$

The use of rFVIIa carries the risk of thrombotic complications. A recent review concluded that the use of rFVIIa increases the risk of arterial (but not venous) thrombosis. ${ }^{23}$ The risk of thrombotic complications in the postpartum patient may be higher compared with other subpopulations.

\section{Proposed Massive Transfusion Protocol}

As previously mentioned, massive transfusion protocols have been adopted widely around the world. Controversy surrounds the optimal ratio of PRBC, FFP, and platelets that needs to be administered. We believe that the improvement in outcomes might not necessarily be the result of a specific ratio administration of blood products, but rather the timely multidisciplinary intervention that is usually triggered when such protocols are activated. Once the obstetrician activates the protocol, everyone on the team, from the nurses to the anesthesiologists, is alerted to the seriousness and critical nature of the situation. Aggressive interventions, such as placement of large-bore peripheral or central lines, arterial lines for blood pressure monitoring, and active warming of the patient rapidly follow. Invariably, the activation of the protocol will improve communication between the surgical team and the blood bank. At the same time, it is not uncommon that, in the hospital setting, sudden occurrence of "emergent complicated cases" leads to the presence of multiple experienced surgeons who will make themselves "available to help" and the activation of backup systems. The direct and indirect effects from activation of the massive transfusion protocol are in stark contrast to the scenario when the seriousness of the hemorrhage is not appreciated by the obstetric provider or not adequately communicated to the ancillary providers. The combination of early recognition and intervention may be more important than a specific administration ratio of blood products. - Table 1 depicts a proposed massive transfusion protocol for use in obstetric practice. Although various guidelines have been proposed for nonobstetric patients, the precise threshold to activate the protocol in obstetric patients is difficult to establish. In practice, the decision is based on the subjective evaluation of the surgeon involved in the case. Criteria that would impact the decision include the estimated blood already lost, the

Table 1 Massive Transfusion Protocol in Obstetrics

\begin{tabular}{|l|l|l|l|l|}
\hline & PRBC & FFP & Platelets & Cryoprecipitate \\
\hline Round 1 & $6 \mathrm{U}$ & $6 \mathrm{U}$ & $6 \mathrm{U}$ & $10 \mathrm{U}$ \\
\hline Round 2 & $6 \mathrm{U}$ & $6 \mathrm{U}$ & $20 \mathrm{U}$ \\
\hline Round 3 & \multicolumn{1}{|l|}{ Recombinant activated factor VII $(40 \mu \mathrm{g} / \mathrm{kg})^{\mathrm{a}}$} & $6 \mathrm{U}$ \\
\hline Round 4 & $6 \mathrm{U}$ & $6 \mathrm{U}$ & & $10 \mathrm{U}$ \\
\hline Round 5 & $6 \mathrm{U}$ & $6 \mathrm{U}$ & \\
\hline Round 6 & \multicolumn{5}{|l}{ Recombinant activated factor VII $(40 \mu \mathrm{g} / \mathrm{kg})$} \\
\hline
\end{tabular}

Adapted with permission from: Pacheco LD, Saade GR, Gei AF, Hankins GD. Cutting-edge advances in the medical management of obstetrical hemorrhage. Am J Obstet Gynecol 2011;205:526-532. ${ }^{4}$

Consider activating the protocol when massive hemorrhage is expected and/or in patients with ongoing bleeding despite receiving $4 \mathrm{U}$ of PRBC within a short period of time (1-2 h). Once activated, blood bank personnel will continue preparing blood products until the protocol is inactivated by the surgical team. After round 6, if not inactivated, the protocol will start again from round 1. PRBC, packed red blood cells; FFO, fresh frozen plasma. aThe utilized dose of recombinant activated factor VII is based on local expert opinion. 
anticipated further blood loss, and the likelihood the bleeding will be controlled any time soon.

We suggest activation of the massive transfusion protocol when hemorrhage is expected to be massive (anticipated need to replace $50 \%$ or more of blood volume within 2 hours) and in patients with ongoing bleeding after the transfusion of $4 \mathrm{U}$ of PRBC within a short period of time (1 to 2 hours). Once activated, the blood bank will prepare and send to the operating room blood products in the order depicted in - Table 1. Blood bank personnel will send the "first round" of products that will include $6 U$ of PRBC, $6 U$ of FFP, $6 U$ of platelets, and $10 \mathrm{U}$ of cryoprecipitate. Unless inactivated, the blood bank will start preparing the products for "round 2" (6 U of PRBCs, 6 U of FFP, and $20 \mathrm{U}$ of cryoprecipitate) and send them to the operating room as soon as they are ready. The sequence will continue until round 6 . If not inactivated, and if the patient still has uncontrolled hemorrhaging, the protocol starts again on round 1 .

\section{Conclusion}

Hemorrhage is the most common form of shock in obstetric practice and remains a major cause of maternal mortality worldwide. Hemostatic resuscitation has emerged as a new concept, albeit based on limited prospective data. Massive transfusion protocols could improve outcomes in the bleeding patient not only due to early blood product administration but also secondarily to an early and aggressive multidisciplinary intervention. Hospitals that institute massive transfusion protocols should ideally include a quality/ peer review committee to guarantee periodic revisions of the available protocol.

\section{Acknowledgments}

For editorial and graphic assistance, we thank Ob/Gyn Publication, Grant, and Media Support director and staff. The office is supported by the Department of Obstetrics and Gynecology at UTMB.

\section{References}

1 Kung HC, Hoyert DL, Xu J, Murphy SL. Deaths: final data for 2005. Natl Vital Stat Rep 2008;56:1-120

2 Kwee A, Bots ML, Visser GH, Bruinse HW. Emergency peripartum hysterectomy: a prospective study in The Netherlands. Eur J Obstet Gynecol Reprod Biol 2006;124:187-192

3 Holcomb JB, Wade CE, Michalek JE, et al. Increased plasma and platelet to red blood cell ratios improves outcome in 466 massively transfused civilian trauma patients. Ann Surg 2008;248: 447-458
4 Pacheco LD, Saade GR, Gei AF, Hankins GD. Cutting-edge advances in the medical management of obstetrical hemorrhage. Am J Obstet Gynecol 2011;205:526-532

5 Rajasekhar A, Gowing R, Zarychanski R, et al. Survival of trauma patients after massive red blood cell transfusion using a high or low red blood cell to plasma transfusion ratio. Crit Care Med 2011;39:1507-1513

6 Nascimento B, Callum J, Rubenfeld G, Neto JB, Lin Y, Rizoli S. Clinical review: Fresh frozen plasma in massive bleedings-more questions than answers. Crit Care 2010;14:202

7 Ickx BE. Fluid and blood transfusion management in obstetrics. Eur J Anaesthesiol 2010;27:1031-1035

8 Glassford NJ, Bellomo R. Acute kidney injury: how can we facilitate recovery? Curr Opin Crit Care 2011;17:562-568

9 Herrler T, Tischer A, Meyer A, et al. The intrinsic renal compartment syndrome: new perspectives in kidney transplantation. Transplantation 2010;89:40-46

10 Yunos NM, Bellomo R, Story D, Kellum J. Bench-to-bedside review: chloride in critical illness. Crit Care 2010;14:226

11 Brohi K, Cohen MJ, Davenport RA. Acute coagulopathy of trauma: mechanism, identification and effect. Curr Opin Crit Care 2007;13:680-685

12 Roberts I, Shakur H, Ker K, Coats T; CRASH-2 Trial collaborators. Antifibrinolytic drugs for acute traumatic injury. (review) Cochrane Database Syst Rev 2011;(1):CD004896

13 Cotton BA, Gunter OL, Isbell J, et al. Damage control hematology: the impact of a trauma exsanguination protocol on survival and blood product utilization. J Trauma 2008;64:1177-1182; discussion 1182-1183

14 Wafaisade A, Maegele M, Lefering R, et al; Trauma Registry of DGU. High plasma to red blood cell ratios are associated with lower mortality rates in patients receiving multiple transfusion $(4 \leq$ red blood cell units $<10$ ) during acute trauma resuscitation. J Trauma 2011;70:81-88; discussion 88-89

15 Snyder CW, Weinberg JA, McGwin G Jr, et al. The relationship of blood product ratio to mortality: survival benefit or survival bias? J Trauma 2009;66:358-362; discussion 362-364

16 Scalea TM, Bochicchio KM, Lumpkins K, et al. Early aggressive use of fresh frozen plasma does not improve outcome in critically injured trauma patients. Ann Surg 2008;248:578-584

17 Stanworth SJ, Birchall J, Doree CJ, Hyde C. Recombinant factor VIla for the prevention and treatment of bleeding in patients without haemophilia. Cochrane Database Syst Rev 2007;(2):CD005011

18 Spahn DR, Cerny V, Coats TJ, et al; Task Force for Advanced Bleeding Care in Trauma. Management of bleeding following major trauma: a European guideline. Crit Care 2007;11:R17

19 Johansson PI, Ostrowski SR, Secher NH. Management of major blood loss: an update. Acta Anaesthesiol Scand 2010;54:1039-1049

20 Bell SF, Rayment R, Collins PW, Collis RE. The use of fibrinogen concentrate to correct hypofibrinogenaemia rapidly during obstetric haemorrhage. Int J Obstet Anesth 2010;19:218-223

21 Gonzalez EA, Moore FA, Holcomb JB, et al. Fresh frozen plasma should be given earlier to patients requiring massive transfusion. J Trauma 2007;62:112-119

22 Gilliss BM, Looney MR, Gropper MA. Reducing noninfectious risks of blood transfusion. Anesthesiology 2011;115:635-649

23 Levi M, Levy JH, Andersen HF, Truloff D. Safety of recombinant activated factor VII in randomized clinical trials. N Engl J Med 2010;363:1791-1800 